\title{
Factors Influencing Utilization of Antenatal Care Services Among Teenage Mothers in Malindi Sub-County Kenya-A Cross Sectional Study
}

\author{
Ndambuki Stephen Mulinge ${ }^{1}$, Oyindamola Bidemi Yusuf ${ }^{2}$, Chris O. Aimakhu ${ }^{3}$ \\ ${ }^{1}$ Department of Reproductive Health, Pan African University, Ibadan, Nigeria \\ ${ }^{2}$ Department of Epidemiology and Medical Statistics, University of Ibadan, Ibadan, Nigeria \\ ${ }^{3}$ Department of Obstetrics and Gynaecology, University College Hospital (UCH), Ibadan, Nigeria \\ Email address: \\ smulinge2010@gmail.com (N. S. Mulinge)
}

\section{To cite this article:}

Ndambuki Stephen Mulinge, Oyindamola Bidemi Yusuf, Chris O. Aimakhu. Factors Influencing Utilization of Antenatal Care Services Among Teenage Mothers in Malindi Sub-County Kenya-A Cross Sectional Study. Science Journal of Public Health. Vol. 5, No. 2, 2017 , pp. $61-67$. doi: $10.11648 /$ j.sjph.20170502.12

Received: December 22, 2016; Accepted: January 9, 2017; Published: January 24, 2017

\begin{abstract}
Effective antenatal care services (ANC) present an opportunity to minimize the high risk for poor perinatal outcomes and other birth complications associated with teenage pregnancy. With the failure by Kenya and majority of the Sub Saharan Countries to achieve the Millennium Development Goals (MDG 4 \& 5), there is need to understand factors influencing utilization of maternal health services among special groups within the reproductive age. Therefore, this study identified factors that influence utilization of ANC services among women aged 13-19 years. A facility-based cross-sectional study was conducted among 385 teenage mothers using semi-structured questionnaires and key informants' interviews in Malindi SubCounty. The WHO recommendation of at least 4 ANC visits was used as standard measure for good or poor ANC services utilization. Quantitative data were analyzed using descriptive statistics, chi square test and logistic regression at the 5\% level of significance while qualitative data were analyzed thematically. Mean age of the participants was $18.10 \pm 1.11$ and about $69 \%$ were married. In total, $91.2 \%$ of the participants reported poor utilization of ANC services with less than 4 ANC visits. About $54.7 \%$ reported late/inappropriate booking time. Knowledge of the recommended ANC visits, marital status, occupation, and the person making the decision to attend ANC were strong determinants of ANC utilization. Participants with good knowledge of the recommended ANC visits were about 3 times more likely to have good utilization of ANC services than participants with poor knowledge $(\mathrm{OR}=2.734, \mathrm{p}=0.006,95 \% \mathrm{CI}$ : 1.34, 5.58).Participants who made the decision to start ANC clinics were 3 times more likely to have good ANC services utilization compared to participants whose decision was made by others $(\mathrm{OR}=2.914, \mathrm{p}=0.009,95 \% \mathrm{CI}: 2.91,6.48)$.Utilization of ANC services among teenage mothers remains significantly poor in Malindi sub-County. Immense efforts should be directed towards creation of awareness about ANC and its importance in the communities, and implementation of innovative programs targeting pregnant teenage girls to encourage them to seek ANC services.
\end{abstract}

Keywords: Teenage Mothers, Antenatal Care, Perinatal Outcomes, Knowledge, Decision

\section{Introduction}

Teenage pregnancy and its associated complications pose a major challenge in efforts to address maternal, infant mortality and related morbidities in Sub-Saharan African countries. Majority of Sub-Saharan African countries including Kenya have fallen short of attaining Millennium Development Goals (MDG) and particularly MDGs 4 and 5 targets [17]. The slow progress in reduction of maternal and infant mortalities can be partially attributed to inequitable access to continuum care before, during, and after pregnancy. According to WHO a maternal mortality ratio in 2013 was estimated to be 230 per 
100,000 live births in developing countries compared to 16 per 100,000 live births in developed countries [1]. Maternal deaths in Kenya were estimated to be about 362 deaths per 100,000 live births by 2014 with large disparities existing across the country with Mandera County reporting extremely high maternal mortality ratios of over 3000 deaths per 100,000 live births [2].

The problem of high child and maternal mortality is exacerbated by the relatively high prevalence of childhood marriages and teenage pregnancies in SSA and the high risks associated with adolescence childbirth. WHO estimates that about 16 million adolescent women give birth each year across the globe with about $95 \%$ of these births in the developing countries [1]. In Kenya, current statistics show that $15 \%$ of women age 15-19 have already had a birth while $18 \%$ have begun child bearing (had a live birth or are pregnant with their first child [3]. WHO further estimates that adolescents (10-19 years), account for about 11 per cent of all global births and as such contribute to about $23 \%$ of pregnancy and childbirth related disease burden worldwide [14]. Strong research-based evidence depicts poorer perinatal outcomes such as preterm births, early neonatal deaths, stillbirth, low birth weight (LBW $<2,500$ at birth) and neonate deaths among the teenage mothers [4].

Teenage pregnancy is associated with an increased risk for stillbirths and neonatal deaths with an estimated 50\% higher risk among women under the age of 20 years compared to older women [1]. Good utilization of the focused ANC services has been shown to minimize the risk for poor perinatal outcomes and other birth complications. However, significant variations in the utilization of ANC services exist among the different groups of women of reproductive age with women aged below 20 years reporting poorer utilization of such services compared to older women [3]. The variations in the utilization and timing of ANC services have been associated with a wide range of factors including lack of knowledge about ANC services and its benefits, the recommended timing for the first $\mathrm{ANC}$ visit, socioeconomic, and demographic factors as well as personal attributes such as perceptions about practitioners and type of pregnancy[5]. Other factors include long distance greater than five kilometers from the facility, the thought that money is required for ANC services, and reported lack of any problem with current pregnancy as a reason for not seeking ANC services especially among high parity women [5].

Nevertheless, few past studies have explored the factors influencing utilization of ANC services among women under the age of 20 years. As such, as the country examines reasons for not realizing the MDG $4 \& 5$ by 2015 , there is need to focus on specific groups within the reproductive age group which potentially undermine efforts towards realization of the various targets [17]. Therefore, this study determined the factors influencing utilization of ANC services among women aged between 13 and 19 years in Malindi Sub-county.

\section{Methodology}

\subsection{Study Area}

Malindi Sub-county is in Kilifi County, and part of the Coastal region of Kenya. The County borders Tana River to the North, TaitaTaveta to the West, Mombasa, and Kwale Counties to the south and the Indian Ocean to the East. Malindi subCounty has a total area of 7, 792 square Kilometers and is further sub-divided into five County Assembly Wards including Malindi town, Kakuyuni, Jilore, Shella, and Ganda. The total population for the sub-county was estimated to be 400,514 people in 2009 while the Kilifi County population has been projected to grow to $1,336,590$ and $1,466,856$ by 2015 and 2017 respectively [6]. The $<15$ population form a significant part of the county's population accounting for $47.5 \%$, youths $19.4 \%$ and age group 25-59 years making up to $28 \%$ [6].

\subsection{Sampling Procedures}

The five County Assembly Wards (CAWs) making up Malindi Sub county were purposively included in the study. A multistage random sampling technique was applied to select public health facilities and the respondents in the study. In the first stage, a sample frame of all public health facilities providing ANC and child wellness services was drawn at the ward level, from which one public health facility was randomly selected by way of balloting. The second stage involved the selection of participants from each ward with the age of the mother identified at the point of care and referred by the health provider to the research assistant for administration of the questionnaires.

\subsection{Sample Size Considerations}

For the purpose of this study, a sample of 385 women who had given birth before the age of 19 years and their child was one year or less old was used.The sample size was determined using the following formulae for single proportions.

(1) $n=\frac{Z \alpha 2 P Q}{d^{2}}$ (Lwanga\&Lemeshow, 1991)

Where $\mathrm{n}$ is the minimum sample size

$\mathrm{Z} \alpha$ is the standard normal deviation corresponding to $95 \%$ confidence level of set at 1.96

$\mathrm{P}$ is the proportion of women below the age of 20 years attending antenatal clinic at the national level (48.9\%) according to KDHS 2014.

$\mathrm{Q}=1-\mathrm{P}$

$\mathrm{d}=$ the desired level of prevision (For this study at $5 \%$ )

$$
\begin{gathered}
\mathrm{n}=\frac{(1.96)^{2} \times(0.489) \times(0.511)}{0.05^{2}} \\
\mathrm{n}=384
\end{gathered}
$$

\subsection{Data Collection}

Data was collected using semi-structured questionnaires administered by trained research assistants and additional information obtained through key informants' interviews. The 
key informant's interviews involved health care providers, Parents, and teenage mothers from each of the wards where the study was conducted.

\subsection{Data Analysis}

Frequency tables and graphs were used to present relevant variables. Descriptive statistics such as means, standard deviations were used to summarize quantitative variables while categorical variables were summarized with percentages. The chi square test was used to investigate associations between selected variables (such as educational level, marital status knowledge level, type of pregnancy), time of booking) and ANC utilization. Variables that were significant in the Chisquare test were entered into a logistic regression model to further investigate the strength of the association model. This was tested with the Hosmer-Lemeshow Goodness-of-Fit Test with the model accepted if the p-value was higher than 0.05 . All analysis were carried out at 5\% level of significance. Qualitative data from the key informants' interviews was analyzed using the thematic approach. For the purpose of this study, $\geq 4$ ANC visits and $<4$ ANC visits was considered good and poor ANC services utilization respectively. Booking time reported within the first 3 months of pregnancy was considered good/appropriate timing.

\section{Results}

Mean age of the participants was 18.10 years $(\mathrm{SD}=1.11)$. Majority of the participants had primary education as the highest level of education attained. About $69 \%$ of the participants were married. Over $50 \%$ of the participants were reported as not working and had no an expected monthly income. In total, $91.2 \%$ had less than $4 \mathrm{ANC}$ visits over their gestational period. There was no statistically significant association between age, level of education religion, expected monthly income, perception of distance to the nearest health care facility and ANC services utilization $(p>0.05)$. Marital status and occupation were found to be associated with utilization of ANC services ( $\mathrm{p}=0.040$ and $\mathrm{p}=0.006$ respectively) (Table. 1).

3.1 ANC Services Utilization by Demographic Characteristics

Table 1. ANC Services Utilization by Demographic Characteristics.

\begin{tabular}{|c|c|c|c|c|c|}
\hline Characteristics & $\geq 4$ Visits & $<4$ Visits & $X^{2}$ & df & P value \\
\hline Age & & & 1.384 & 2 & 0.500 \\
\hline 13-17 years & $6(6.0 \%)$ & $94(94.0 \%)$ & & & \\
\hline $18-19$ years & $26(9.9 \%)$ & $236(90.1 \%)$ & & & \\
\hline 20 years & $2(8.7 \%)$ & $21(91.3 \%)$ & & & \\
\hline Education Level & & & 7.195 & 3 & 0.066 \\
\hline Primary level & $22(8.8 \%)$ & $227(91.2 \%)$ & & & \\
\hline Secondary level & $6(5.6 \%)$ & $102(94.4 \%)$ & & & \\
\hline Tertiary level & $4(23.5 \%)$ & $13(76.5 \%)$ & & & \\
\hline Not Been to School & $2(18.2 \%)$ & $9(81.8 \%)$ & & & \\
\hline Marital Status & & & 4.214 & 1 & 0.040 \\
\hline Single & $5(4.3 \%)$ & $111(95.7 \%)$ & & & \\
\hline Married & $29(10.8 \%)$ & $240(89.2 \%)$ & & & \\
\hline Religion & & & 0.762 & 2 & 0.683 \\
\hline Christian & $20(8.0 \%)$ & $230(92.0 \%)$ & & & \\
\hline Muslim & $12(10.8 \%)$ & $99(89.2 \%)$ & & & \\
\hline Other & $2(8.3 \%)$ & $22(91.7 \%)$ & & & \\
\hline Occupation & & & 10.189 & 2 & 0.006 \\
\hline Casual laborer & $3(4.3 \%)$ & $66(95.7 \%)$ & & & \\
\hline Business Woman & $10(20.4 \%)$ & $39(79.6 \%)$ & & & \\
\hline Not Working & $21(7.9 \%)$ & $246(92.1 \%)$ & & & \\
\hline
\end{tabular}

\subsection{Booking Time}

In total, $71(18.3 \%)$ of the participants reported their booking time or first ANC visit during the first trimester, 157 (40.8\%) during the $2^{\text {nd }}$ trimester, and $54(13.9 \%)$ during 3rd trimester, whereas $103(26.5 \%)$ did not know the gestational age when they reported for their first visit. Booking time was associated with the type of pregnancy and the person making the decision to attend ANC (table. 2). Those who made the decision to attend ANC clinic were three times likely to report appropriate booking compared to participants whose decision to attend ANC clinic was made by others $(\mathrm{OR}=2.717,95 \% \mathrm{CI}$ : $0.033,0.241)$. 
Table 2. Association of Booking Time and Selected Barriers.

\begin{tabular}{|c|c|c|c|c|c|}
\hline \multirow[t]{2}{*}{ Selected Variable } & \multicolumn{3}{|c|}{ Booking Time } & \multirow[t]{2}{*}{$\mathbf{X}^{2}$} & \multirow[t]{2}{*}{ P-value } \\
\hline & First Trimester & $2^{\mathrm{ND}}$ Trimester & Third Trimester & & \\
\hline Typeof Pregnancy & & & & 78.777 & 0.0001 \\
\hline Planned & $28(36.40 \%)$ & $24(31.20 \%)$ & $4(5.2 \%)$ & & \\
\hline Unplanned & $43(14.0 \%)$ & $133(43.20 \%)$ & $50(16.20 \%)$ & & \\
\hline Person Making Decision & & & & 14.678 & 0.023 \\
\hline Myself & $19(35.20 \%)$ & $19(35.20 \%)$ & $5(9.30 \%)$ & & \\
\hline Others & $52(15.80 \%)$ & $138(41.80 \%)$ & $49(14.80 \%)$ & & \\
\hline
\end{tabular}

From the qualitative results the health care providers as the key informants reported that, "The young girls don't understand why they have to come for the clinics and have to be pushed by parents." A teenage mother reported that, "My mother did not want people to know I was pregnant." (KII). 3.3 Knowledge about ANC Services

Majority of the participants, 349 (90.6\%) considered collection of ANC clinic cards as one of the reasons attending ANC. Birth planning was considered a reason for attending ANC among $63 \%$ of the participants. Collection of mosquito nets was highly considered (98\%) as one of the reasons the participants went for ANC. In total, $83 \%$ of the participants did not consider being booked for caesarian section, a reason for attending ANC. Checking for the sex of the baby was not considered as a reason for attending ANC among $80 \%$ of the participants.3.4 Knowledge of the Recommended Minimum ANC Visits and Appropriate Booking Time

In total, $111(29 \%)$ of the participants had good knowledge of the recommended minimum number of ANC clinic visits over a pregnancy period and $274(71 \%)$ had poor knowledge. About 91 (24\%) had good knowledge of the appropriate booking time and 294 (76\%) had poor (incorrect) knowledge of the appropriate booking time.

In the qualitative data, health providers indicated that most teenage mothers do not understand the required number of ANC visits and the appropriate booking time. "Majority of the teenage mothers lack information about ANC." (Health Provider)

"I didn't know when to start." (Teenage mother)

"They don't know when to start, they keep on postponing." (Parent)

\subsection{Knowledge of Danger Signs During Pregnancy}

In total $94.5 \%$ of the participants agreed that bleeding or fluids leaking from the vagina was a danger sign during pregnancy, $50.6 \%$ agreed blurring or sudden impaired vision was a danger sign while $49.4 \%$ disagreed. Sudden stomach pains or backaches was agreed to be a danger sign among $85 \%$ of the participants while $15 \%$ disagreed. About $80.3 \%$ of the participants agreed that frequent, severe, or constant headaches were a danger sign and $19.7 \%$ disagreed. Swelling of limbs was considered a danger sign among $65.5 \%$ of the participants while $34.5 \%$ of the participants did not consider that a danger sign.

\subsection{Barriers to ANC Services Utilization}

Majority of the participants reported their previous pregnancy as unplanned. The type of pregnancy was found to have a statistically significant association with the booking time $(\mathrm{p}<0.0001)$. Over $80.0 \%$ of the participants reported the decision to attend ANC to have been made by another person other than them. Participants who made the decision to start ANC clinics themselves were three times more likely to have good ANC services utilization compared to participants whose decision was made others $(\mathrm{OR}=2.914, \mathrm{p}=0.009,95 \%$ CI: $2.914,6.481)$. Majority of the participants $(54.7 \%)$ reported that they were uncomfortable receiving ANC services together with older women. Analysis of the Likert scale responses on perception of quality of services revealed that most participants (over 50\%) were satisfied with the quality of ANC services provided. In total, 67 (17.4\%) reported that they were charged a fee at the ANC clinic and $302(78.4 \%)$ were not asked to pay any fee. About 308 $(80.2 \%)$ of the participants indicated the amount paid was not expensive and $13(3.4 \%)$ as expensive.

\subsection{Misconceptions About ANC}

Majority $(90.0 \%)$ of the participants dispelled some of misconception about ANC services. About $62.4 \%$ of the participants indicated that the misconception that young girls are insulted/rebuked by nurses during ANC while 37.3\% considered the misconception as true. Majority, (97.2\%) refuted the misconception that $\mathrm{HIV}$ is tested by force and results shared with family members during ANC.

3.6 Association of Selected Variables with ANC Services Utilization

Good utilization of ANC services ( $\geq 4$ ANC visits) was associated with good knowledge of the recommended minimum ANC visits $(p=0.004)$. On the other hand participants with good knowledge of the appropriate booking time $(13.2 \%$ reported better ANC services utilization compared to participants $(7.5 \%)$ with poor knowledge of the appropriate booking time. However, the difference was found not to be statistically significant $(p=0.094)$. Making the decision to attend ANC was associated with utilization of ANC services with participants who made the decision to attend ANC themselves (18.5\%) having higher ANC utilization than those whose the decision was made by others $(7.3 \%)(p=0.025)$. Appropriate booking time was associated with utilization of ANC services among the participants $(p=0.022)$. There was no association between planned and unplanned pregnancy and ANC services utilization 
$(\mathrm{p}=0.151)$. In addition, there was no statistically significant association between perception of the quality of care, and waiting time at the clinic and the ANC services utilization. (Table 3)

Table 3. Association of Selected Variables with ANC Utilization.

\begin{tabular}{|c|c|c|c|c|c|}
\hline & & ANC Utilization & & & \\
\hline Variables & $\geq 4$ Visits & $<4$ Visits & $\mathrm{X}^{2}$ & df & p-value \\
\hline Knowledge of Recommended ANC Visits & & & 8.145 & 1 & 0.004 \\
\hline Good knowledge & $17(15.3 \%)$ & $94(84.7 \%)$ & & & \\
\hline Poor Knowledge & $17(6.2 \%)$ & $257(93.8 \%)$ & & & \\
\hline Knowledge of Appropriate Booking Time & & & 2.808 & 1 & 0.094 \\
\hline Good Knowledge & $12(13.2 \%)$ & $79(86.8 \%)$ & & & \\
\hline Poor Knowledge & $22(7.5 \%)$ & $272(92.5 \%)$ & & & \\
\hline \multicolumn{6}{|l|}{ Type of Pregnancy } \\
\hline Planned & $10(13.0 \%)$ & $67(87.0 \%)$ & 2.065 & 1 & 0.151 \\
\hline Unplanned & $24(7.8 \%)$ & $284(92.2 \%)$ & & & \\
\hline Person making decision to attend ANC & & & 7.387 & 2 & 0.025 \\
\hline Myself & $10(18.5 \%)$ & $44(81.5 \%)$ & & & \\
\hline Others & $24(7.3 \%)$ & $306(92.7 \%)$ & & & \\
\hline Perception of Quality of Care Received & & & 4.671 & 3 & 0.198 \\
\hline Good quality & $28(11.1 \%)$ & $225(88.9 \%)$ & & & \\
\hline Poor quality & $5(4.3 \%)$ & $110(95.7 \%)$ & & & \\
\hline Perception of the Waiting Time & & & 3.882 & 2 & 0.144 \\
\hline Short & $20(12.1 \%)$ & $145(87.9 \%)$ & & & \\
\hline Long & $13(6.4 \%)$ & $191(93.6 \%)$ & & & \\
\hline Booking Time & & & 7.634 & 2 & 0.022 \\
\hline Appropriate timing & $9(12.7 \%)$ & $62(87.3 \%)$ & & & \\
\hline Poor Timing & $11(5.2 \%)$ & $200(94.8 \%)$ & & & \\
\hline
\end{tabular}

\subsection{Determinants of ANC Services Utilization}

Participants with good knowledge of the recommended minimum ANC visits were 3 times more likely to have good utilization of ANC services ) than participants with poor knowledge $(\mathrm{OR}=2.734, \mathrm{p}=0.006,95 \%$ CI 1.341, 5.58). Participants with appropriate booking time were once more likely to report good utilization of ANC services compared to those with poor knowledge. However, this was found not to be statistically significant $(\mathrm{OR}=1.084, \mathrm{p}=0.081,95 \% \mathrm{CI}: 0.44$, 2.66). Marital status was found to influence utilization of ANC services with married participants 3 times likely to have good utilization of ANC services compared to the single participants ( $\mathrm{OR}=2.682, \mathrm{P}=0.047,95 \% \mathrm{CI}: 0.14,0.99)$. Participants who made the decision to start ANC clinics were 3 times more likely to have good ANC services utilization compared to participants whose decision was made by others $(\mathrm{OR}=2.914$, $\mathrm{p}=0.009,95 \%$ CI: $2.914,6.481)$. Participants who reported their occupation as businesswomen were $67 \%$ less likely to have good ANC services utilization compared to casual laborers and those not working. $(\mathrm{OR}=0.333, \mathrm{p}<0.009,95 \% \mathrm{CI}$ : $0.146,0.760)$.

Table 4. Logistic Regression Results.

\begin{tabular}{|c|c|c|c|c|c|}
\hline Variables & $\mathbf{O . R}$ & S.E & P-value & Upper & 95\% CILower \\
\hline \multicolumn{6}{|l|}{ Knowledge of the Recommended ANC Visits } \\
\hline Good knowledge & 2.734 & 0.364 & 0.006 & 1.341 & 5.575 \\
\hline \multicolumn{6}{|l|}{ Poor Knowledge } \\
\hline Booking Time & 1.084 & 0.458 & 0.861 & 0.441 & 2.660 \\
\hline \multicolumn{6}{|l|}{ Appropriate booking time } \\
\hline \multicolumn{6}{|l|}{ Inappropriate booking time } \\
\hline Marital Status & 2.682 & 0.498 & 0.047 & 1.011 & 7.114 \\
\hline \multicolumn{6}{|l|}{ Married } \\
\hline \multicolumn{6}{|l|}{ Single } \\
\hline Person Making the Decision to Attend ANC & 2.914 & 0.408 & 0.009 & 2.914 & 6.481 \\
\hline \multicolumn{6}{|l|}{ Myself } \\
\hline \multicolumn{6}{|l|}{ Others } \\
\hline \multicolumn{6}{|l|}{ Occupation } \\
\hline Casual laborer & 1.878 & 0.993 & 0.319 & 0.544 & 6.489 \\
\hline Business women & 0.333 & 0.421 & 0.009 & 0.146 & 0.760 \\
\hline
\end{tabular}




\section{Discussion}

Antenatal care (ANC) is one of the critical elements of the continuum care that women should receive in order to avert the wide range of risks associated with pregnancy. Effective utilization of ANC services has been demonstrated to improve pregnancy outcomes and subsequently minimize risks associated with childbirth. WHO recommends the focused ANC approach entail at least four ANC visits as and effective strategy for ensuring that women receive the essential ANC services during the gestational period. However, studies and reports have reported disparities in utilization of ANC services among different groups of women with the reproductive age [15].

It was estimated that about $22 \%$ of the women in Malindi Sub-county begin child bearing before the age of 19 years [3]. Our study findings agree with this; mean age of the participants in this study was also 19 years. The apparently high number of childbirths before the age of 19 years could be attributed to low contraceptive use, which currently stands at 20\% among married girls aged 15-19 who, use modern contraceptives, considerably lower than $37 \%$ at the national level [7]. Our study further established that majority of the participants had only attained primary level education. This can be attributed to low primary to secondary school transition rate [8].

Our study found that majority of the participants were married, a finding supported by the KDHS 2014 report in which half of Kilifi County women aged 25-49 years, got married by the age of 19 years [7]. Majority of the participants were also not working and had no expected monthly income. This is because majority of the teenage mothers drop out of school and lack employability skills, a phenomenon that explains the finding that majority of them are not working or are engaged in casual work [9].

Our study found out that as low as $8.8 \%$ of the teenage mothers reported the WHO recommended $\geq 4 \mathrm{ANC}$ visits and a large majority had less than $4 \mathrm{ANC}$ visits. The percentage of teenage mothers attending at least 4 ANC clinics was too low compared to that reported for women aged below 20 years at the national level [3]. The findings are similar to other research studies conducted in different parts of Sub- Saharan Africa in which women aged 15-19 years report poor utilization of ANC services $[5,10]$. Although other studies have found significant influence of education level on ANC services utilization among women of reproductive age [11], our study did not show a statistically significant association between level of education and ANC services utilization.

The study found knowledge of the recommended ANC visits, marital status, occupation, and the person making the decision to attend ANC to be strong determinants of ANC utilization among teenage mothers. Participants with good knowledge of the recommended minimum ANC visits were more likely to have good utilization of ANC services compared to participants with poor knowledge. Married participants were also more likely to have good utilization of ANC services compared to the single participants. Marital status can have both positive and negative effects on the utilization of ANC services as reported in different studies with uneducated husbands resulting into poor timing and utilization of ANC services $[12,13]$.

Those who made the decision to attend ANC clinic were three times likely to report appropriate booking compared to participants whose decision to attend ANC clinic was made by others. Responses from the key informants revealed that in many cases teenage mothers wait to be pushed by parents or guardians to attend ANC. However, the interviews also found that most parents do not have adequate knowledge about ANC services and particularly the appropriate timing time, required number of ANC visits and the benefits.

Our study established that majority of the teenage mothers do not have adequate knowledge about important aspects of ANC services including the appropriate booking time and the recommended number of visits. This translated into majority reporting inappropriate or late booking and less than the recommended 4 ANC visits. However, there was a general good knowledge of the common danger signs during pregnancy and majority of the participants dispelled common misconceptions about ANC services. This can be attributed to improved awareness of about ANC services in the country as shown by the high percentage of women reporting at least one ANC visit. Evince

According to the study findings, majority of the participants perceived the quality of service provided during ANC clinics as good. However, most of the participants were not comfortable receiving ANC services together with older women. The findings were further strengthened by responses from the key informants' interviews in which those interviewed reported that teenage mothers fear judgment and ridicule from older women.

\section{Conclusion}

As the problem of teenage pregnancy continues to persist in most sub-Saharan countries, significant effort is needed to ensure improved access to maternal health services such as antenatal care. The study established that majority of the teenage mothers attend at least one ANC visit but report less than the recommended 4 ANC visits during their pregnancy. Notably, majority of the participants lacked knowledge about the appropriate booking time and the required minimum number of ANC visits. According to the study findings, poor utilization of ANC services can be attributed to several factors including lack of knowledge about the appropriate booking time and the minimum ANC visits, the perception that money is required for the services, fear or shyness, discomfort in attending ANC together with older women and the fact that majority of the teenage girls do not make the decision themselves to attend ANC themselves. As such efforts to address the problem of poor utilization of ANC services among teenage mothers should be focused on creation of awareness about key aspects of ANC services such as appropriate booking time, recommended minimum visits over the gestational period, 
benefits of ANC service utilization, and empowerment of teenage mothers. However, different stakeholders need to acknowledge prevention of teenage pregnancies as the only sure means to minimize the risks associated with premature childbirth.

\section{References}

[1] WHO. (2015). Maternal mortality. Retrieved fromhttp://www.who.int/mediacentre/factsheets/fs348/en/.

[2] UNFPA, Kenya. (2014). Counties with the highest burden of maternal mortality. News, 2014. Retrieved September 4, 2015, http://countryoffice.unfpa.org/kenya/2014/08/13/10333/counties _with_the_highest_burden_of_maternal_mortality/.

[3] Kenya Demographic and Health Survey (2014). Key Indicators. Retrieved August 22, 2015.

[4] Althabe, F. et al. (2015). Adverse maternal and perinatal outcomes in adolescent pregnancies: The Global Network's Maternal Newborn Health Registry study. Reproductive Health $12(2) 1-9$.

[5] Kisuule, I. et al. (2013). Timing and reasons for coming late for the first antenatal care visits by pregnant women at Mulango, Kampala Uganda. BMC Pregnancy and Childbirth 13 (12), 11-7.

[6] Kenya Inter-Agency Rapid Assessment. (2014). Kilifi Secondary Data Review as at February 2014. KIRA. Retrieved from https://www.humanitarianresponse.info/system/files/documents/ files/Kilifi\%20Secondary\%20Data\%20Review_20141112.pdf.

[7] African Institute for Development Policy (AFIDEP). (2016). Adolescent sexual and Reproductive health in Kilifi County: Fact Sheet. Ministry of Health, Print.

[8] Kiptanui, C., Kindiki, J \& Lelan, J. (2015). Impact of teenage motherhood on the academic performance in public primary schools in Bungoma County, Kenya. International Journal of Educational Administration and Policy, 7 (2), 61-71.
[9] Leerlooijer, J et al. (2014). Applying intervention mapping to develop a community-based intervention aimed at improved psychological and social well-being of unmarried teenage mothers in Uganda. Health Education Research, 31 (4) 1-13.

[10] Rai, RK., Singh, PK \& Singh, L. (2012). Utilization of maternal health care services among married adolescent women: Insight from the Nigerian Demographic and Health Survey, 2008. Women's Health Issues 22 (4). 407-414.

[11] Mabitsela, O. (2010). Adolescent health service in South Africa. The National Adolescents Friendly Clinic Initiative. Retrieved from http://www.ppdafrica.org/docs/southafricaadolescent.pdf.

[12] Dairo, M, D \& Owoyukun, K. E. (2010). Factors affecting utilization of antenatal care servicesin Ibadan, Nigeria. 12 (1), 413.

[13] Nansubuga, R. (2011). Factors affecting the utilization of antenatal care services among adolescent pregnant mothers. A case study of Naguru Teenage Health Center, Kampala, Uganda. Proc. $58^{\text {th }}$ World Statistical Congress, Bublin.

[14] Muyunda, B., Makasa, M., Jacobs, C., Musonda, P \& Michelo, C. (2016). Higher education attainment association with optimal antenatal care visits among childbearing women in Zambia. Frontiers in Public Health. Retrieved from http://journal.frontiersin.org/article/10.3389/fpubh.2016.00127/f ull.

[15] Ochako, R., Fotso, J., Ikamari, L., \& Khasakhala, A. (2011). Utilization of maternal health services among young women in Kenya: Insights from the Kenya Demographic and Health Survey, 2003. BMC Pregnancy and Childbirth 11 (1), 1-9.

[16] Wamalwa, E. (2015). Implementation challenges of free maternity services policy in Kenya: The health workers' perspective. The Pan African Medical Journal, 22 (375), 1-5.

[17] Ministry of Devolution and Planning. (2013). Millennium Development Goals. Status Report for Kenya. 